Phot oemi ssi on and band- cal cul at i on st udi es of the charge- densi ty wave in CuV2S4

\begin{tabular}{|l|l|}
\hline 著者 & $\begin{array}{l}\text { MATSUNO J., FUJI MORI A., MATTHEI SS L. F. , } \\
\text { ENDO R. , NAGATA Shoi chi }\end{array}$ \\
\hline $\begin{array}{l}\text { j our nal or } \\
\text { publ i cat i on ti tl e }\end{array}$ & $\begin{array}{l}\text { Physi cal revi ew. Thi rd ser i es. B, Condensed } \\
\text { nat ter and mat er i al s physi cs }\end{array}$ \\
\hline vol une & 64 \\
\hline number & 11 \\
\hline page r ange & 115116-1-115116- 4 \\
\hline year & 2001- 09-15 \\
\hline URL & ht t p: //hdl . handl e. net /10258/247 \\
\hline
\end{tabular}




\title{
Photoemission and band-calculation studies of the charge-density wave in $\mathrm{CuV}_{2} \mathrm{~S}_{4}$
}

\author{
J. Matsuno, ${ }^{1}$ A. Fujimori, ${ }^{1,2}$ and L. F. Mattheiss ${ }^{1}$ \\ ${ }^{1}$ Department of Physics, University of Tokyo, Bunkyo-ku, Tokyo 113-0033, Japan \\ ${ }^{2}$ Department of Complexity Science and Engineering, University of Tokyo, Bunkyo-ku, Tokyo 113-0033, Japan \\ R. Endoh and S. Nagata \\ Department of Materials Science and Engineering, Muroran Institute of Technology, Muroran, Hokkaido 050-8585, Japan
}

(Received 9 March 2001; published 31 August 2001)

\begin{abstract}
We have studied the electronic structure and its changes across the charge-density-wave (CDW) transition in spinel-type $\mathrm{CuV}_{2} \mathrm{~S}_{4}$ by photoemission spectroscopy and first-principles band-structure calculations. The photoemission spectra show pseudogap-like behavior and the gap size is estimated to be $\sim 90 \mathrm{meV}$. The large energy scale of the pseudogap compared to the transition temperature and its anomalous temperature dependence implies that the involved interaction is in the strong-coupling regime. The calculated electronic susceptibility $\chi(\mathbf{q})$ shows a small peak at $\mathbf{q} \simeq \frac{11}{40}[110]$, which is consistent with the observed wave vector $\mathbf{q}$ $\simeq \frac{1}{4}[110]$ characterizing the CDW. This result suggests that Fermi surface nesting is at least partly responsible for the CDW formation.
\end{abstract}

DOI: 10.1103/PhysRevB.64.115116

PACS number(s): 71.45.Lr, 79.60.Bm, 71.20.-b

\section{INTRODUCTION}

Recently, a number of studies have been devoted to quasione-dimensional materials. From a theoretical point of view, one dimensionality leads to a lot of anomalous properties which differ qualitatively from higher-dimensional materials. In particular, it is well known that the metallic state is unstable with respect to the lattice distortion which forms an insulating ground state. Such an instability, called Peierls instability, in one-dimensional materials is often accompanied by the formation of a charge-density wave (CDW) with the same wave vector.

In three-dimensional systems, on the contrary, only a few materials have been reported to show CDW's. Among them, a spinel-type compound $\mathrm{CuV}_{2} \mathrm{~S}_{4}$ is known to undergo a phase transition accompanied by a CDW at $90 \mathrm{~K} .{ }^{1}$ It shows the presence of an incommensurate superlattice with wave vector $\mathbf{q}=\left(\frac{1}{4}-\delta\right)[110]$ at $90 \mathrm{~K}$, which gradually locks in into a commensurate one $\frac{1}{4}[110]$ at $75 \mathrm{~K}$. At $50 \mathrm{~K}$, the wave vector abruptly changes from $\frac{1}{4}[110]$ to $\left(\frac{1}{3}-\delta\right)[110]$. At room temperature, $\mathrm{CuV}_{2} \mathrm{~S}_{4}$ has a normal spinel structure, in which the $\mathrm{Cu}$ ion is tetrahedrally coordinated and the $\mathrm{V}$ ion is octahedrally coordinated by sulphur atoms. The symmetry at lower temperature has been controversial; an electron diffraction study has shown orthorhombic symmetry, ${ }^{2}$ whereas $\mathrm{x}$-ray diffraction has shown cubic ${ }^{1}$ or tetragonal ${ }^{3}$ symmetry. The magnetic susceptibility is independent of temperature above $90 \mathrm{~K}$ and shows sudden decrease at $90 \mathrm{~K}^{3-5}$ The linewidth of the ${ }^{51} \mathrm{~V}$ NMR spectrum abruptly increases below $90 \mathrm{~K}$, indicating the existence of the CDW. ${ }^{6,7}$ Electrical resistivity shows an anomalous upturn below $90 \mathrm{~K} .{ }^{4,5,8} \mathrm{X}$-ray photoemission and $\mathrm{x}$-ray emission spectroscopy studies by $\mathrm{Lu}$ et al. ${ }^{9}$ have shown that the $\mathrm{Cu}$ ion is in the monovalent state. Hence, the $\mathrm{Cu} 3 d$ electrons hardly contribute to the thermodynamic and transport properties and the $\mathrm{V} 3 d$ electrons mainly participate in them. They have also reported first-principles band-structure calculations and estimated the electron-phonon coupling parameter $\lambda$ to be rather large, $\sim 2.0$. Because of its three-dimensional crystal structure, it is unlikely that $\mathrm{CuV}_{2} \mathrm{~S}_{4}$ shows a perfect nesting, but an imperfect nesting effect like the spin-density wave (SDW) in chromium metal ${ }^{10}$ remains possible.

Photoemission spectroscopy has been utilized for the study of many CDW systems in the last decade because of its advantage of directly probing the CDW gap. ${ }^{11}$ In this paper, we present a photoemission study of $\mathrm{CuV}_{2} \mathrm{~S}_{4}$, emphasizing the detailed temperature dependence of the spectra. Spectra of $\mathrm{CuTi}_{2} \mathrm{~S}_{4}$, which is a paramagnetic metal down to $0.5 \mathrm{~K}^{12}$ are also presented for comparison. We have also performed first-principles band-structure calculations in order to obtain insight into the possibility of CDW formation due to Fermi surface nesting.

\section{EXPERIMENT}

Single crystals of $\mathrm{CuV}_{2} \mathrm{~S}_{4}$ and $\mathrm{CuTi}_{2} \mathrm{~S}_{4}$ were synthesized in a quartz ampoule by $\mathrm{TeCl}_{4}$ vapor transport at growth temperatures from 830 to $720^{\circ} \mathrm{C}$ in a two-zone furnace. The $\mathrm{X}$-ray diffraction pattern confirmed that the pure-spinel phase was obtained. ${ }^{4}$

Ultraviolet photoemission (UPS) measurements were made using the He I line $(h \nu=21.2 \mathrm{eV})$. The measurements were performed at various temperatures between $30 \mathrm{~K}$ and $340 \mathrm{~K}$ in order to study spectral changes above and below the transition temperature. In order to calibrate binding energies and estimate the instrumental resolution, gold was evaporated on the sample surface after each series of measurements. The resolution was estimated to be $34 \mathrm{meV}$. The samples were repeatedly scraped in situ with a diamond file. The base pressure of the spectrometer was $\sim 5 \times 10^{-10}$ Torr.

\section{RESULTS AND DISCUSSION}

\section{A. Photoemission spectroscopy}

Figure 1(a) shows valence-band spectra of $\mathrm{CuV}_{2} \mathrm{~S}_{4}$ and $\mathrm{CuTi}_{2} \mathrm{~S}_{4}$. While the overall features of the spectra are similar 

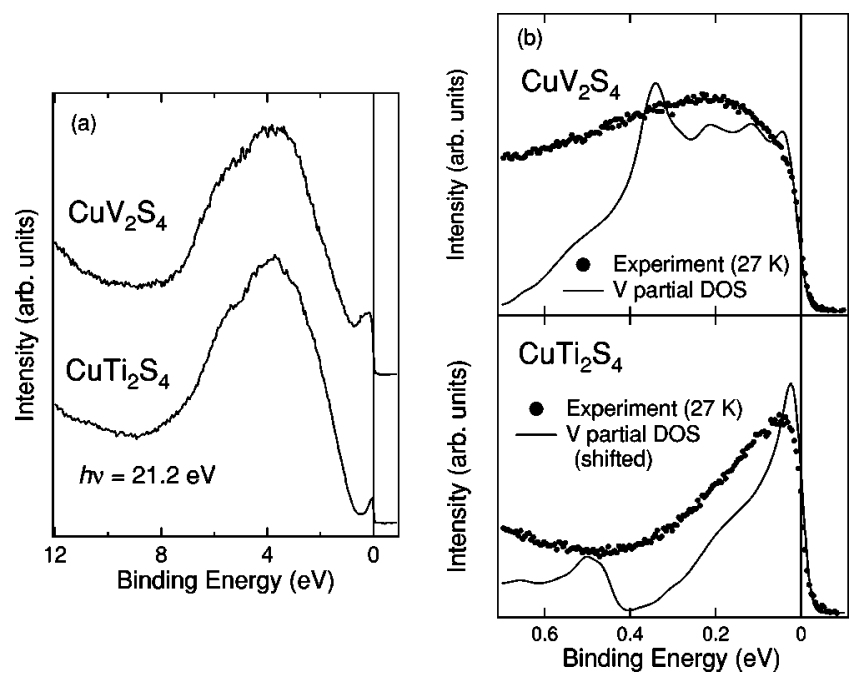

FIG. 1. (a) Photoemission spectra of $\mathrm{CuV}_{2} \mathrm{~S}_{4}$ and $\mathrm{CuTi}_{2} \mathrm{~S}_{4}$ taken at $27 \mathrm{~K}$. The spectra have been normalized to the total integrated intensity. (b) Comparison between the experimental and theoretical spectra for $\mathrm{CuV}_{2} \mathrm{~S}_{4}$ (upper panel) and $\mathrm{CuTi}_{2} \mathrm{~S}_{4}$ (lower panel). Solid lines are the broadened $\mathrm{V} d$ partial density of states. The spectra have been normalized to the integrated intensity between $0.1 \mathrm{eV}$ to $E_{F}$ for each material.

to each other, there are significant differences between the spectra near the Fermi level $\left(E_{F}\right)$. The spectra near $E_{F}$ mainly consist of emission from $\mathrm{V} 3 d / \mathrm{Ti} 3 d$ electrons and the difference reflects the number of $d$ electrons, i.e., $\mathrm{V} 3 d^{1.5}$ versus Ti $3 d^{0.5}$

Figure 1(b) shows a comparison between the measured spectra and theoretical photoemission spectra in the vicinity of $E_{F}$. To obtain the theoretical photoemission spectra, the $\mathrm{V}$ $d$ partial density of states (DOS) calculated in the present work has been convoluted with a Gaussian of $34 \mathrm{meV}$ full width at half maximum (FWHM). For $\mathrm{CuTi}_{2} \mathrm{~S}_{4}$, we rigidly shifted the Fermi level so that the occupied number of states per transition-metal atom becomes one fewer than that in $\mathrm{CuV}_{2} \mathrm{~S}_{4}$. Agreement between the experimental and theoretical spectra is fairly good for both $\mathrm{CuV}_{2} \mathrm{~S}_{4}$ and $\mathrm{CuTi}_{2} \mathrm{~S}_{4}$. In the oxides of early transition metals [for example, $\mathrm{VO}_{2}$ (Ref. 13)], electron correlation within the $3 d$ band is generally strong enough to result in a strong discrepancy between the band-structure calculation and the photoemission spectra. On the other hand, the discrepancy is much smaller in the sulfides of early transition metals. ${ }^{14,15}$ In accordance with those studies, our result suggests that electron correlation has a relatively small effect on the electronic structure of $\mathrm{CuV}_{2} \mathrm{~S}_{4}$.

The upper panels of Fig. 2 show photoemission spectra of $\mathrm{CuV}_{2} \mathrm{~S}_{4}$ and $\mathrm{CuTi}_{2} \mathrm{~S}_{4}$ near $E_{F}$ taken at 27 and $300 \mathrm{~K}$. If the DOS of quasiparticles is independent of temperature, the observed temperature dependence can be explained solely by that of the Fermi-Dirac distribution function. Then the spectral weight between $\sim 4 k_{B} T$ below $E_{F}$ and $\sim 4 k_{B} T$ above it should be conserved. This is the case for $\mathrm{CuTi}_{2} \mathrm{~S}_{4}$, while for $\mathrm{CuV}_{2} \mathrm{~S}_{4}$ the spectral weight increases with temperature just above $E_{F}$ but is almost independent of temperature just below $E_{F}$. To eliminate the temperature dependence of the Fermi-Dirac distribution function, we have divided the pho-
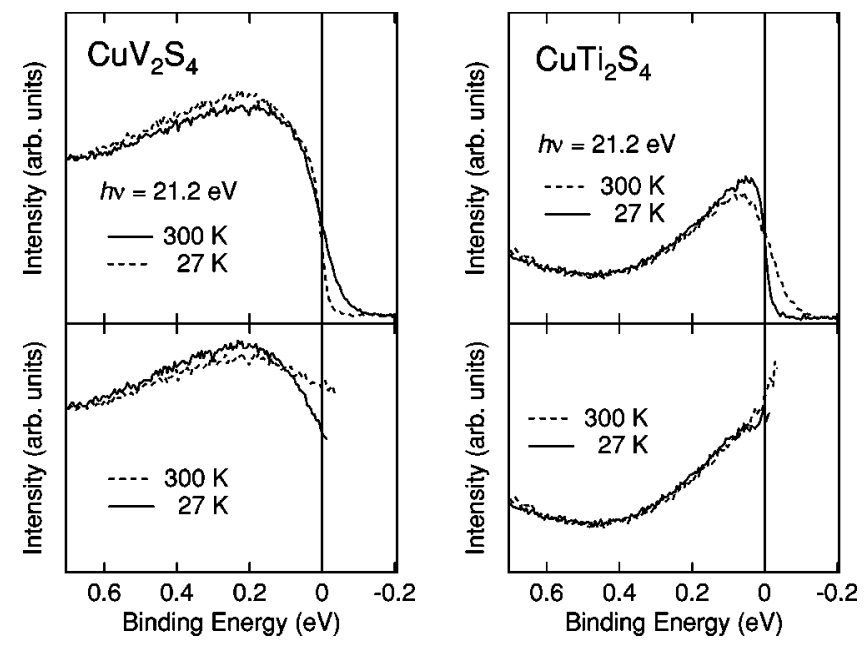

FIG. 2. Upper panels: photoemission spectra of $\mathrm{CuV}_{2} \mathrm{~S}_{4}$ and $\mathrm{CuTi}_{2} \mathrm{~S}_{4}$ near the Fermi level taken at $30 \mathrm{~K}$ and $300 \mathrm{~K}$. The spectra have been normalized to the integrated intensity between $1.3 \mathrm{eV}$ and $E_{F}$ for each material. Lower panels: photoemission spectra with the Fermi-Dirac distribution divided out. See text for detail.

toemission spectra by the Fermi-Dirac distribution function (convoluted with a Gaussian corresponding to the instrumental resolution). ${ }^{16}$ The obtained spectral DOS is shown in the lower panels of Fig. 2. The DOS is clearly dependent on temperature for $\mathrm{CuV}_{2} \mathrm{~S}_{4}$ and the spectral weight below $E_{F}$ to $\sim 100 \mathrm{meV}$ is transferred to higher binding energies with temperature. In $\mathrm{CuTi}_{2} \mathrm{~S}_{4}$, on the contrary, the DOS is nearly independent of temperature. Therefore, it seems that the observed temperature dependence of the spectra of $\mathrm{CuV}_{2} \mathrm{~S}_{4}$ reflects the change in the electronic structure associated with the CDW formation.

A more detailed temperature dependence of the spectra and spectral DOS of $\mathrm{CuV}_{2} \mathrm{~S}_{4}$ is presented in Figs. 3(a) and 3 (b), respectively. All the spectra cross near $100 \mathrm{meV}$ and the spectral weight transfer between $<100 \mathrm{meV}$ and $>100 \mathrm{meV}$ is again observed. The temperature-dependent change in the spectra can be described by the opening of a pseudogap, the size of which is $\sim 100 \mathrm{meV}$. When part of the Fermi surface is nested for the wave vector $\mathbf{q}$, a CDW gap will open only in the nested part of the Fermi surface. This picture is consistent with our result that the gap does not completely open at $E_{F}$. On the other hand, the temperaturedependent change continues to exist up to $>300 \mathrm{~K}$, apparently different from the BCS behavior, in which the gap opens only below $T_{C}$. In order to clarify this point, we have estimated the size of the pseudogap from the midpoint of the initial slope, which is plotted as a function of temperature in Fig. 3(c). The energy at which the DOS takes its maximum is also shown as another measure of the gap magnitude. Both of them are almost independent of temperature and they do not close even at $340 \mathrm{~K}$, although the gap estimated by the dip depth decreases slightly with temperature. The gap energy of $\sim 90 \mathrm{meV}$ is considerably larger than the value $\Delta$ of $14 \mathrm{meV}$ expected from the BCS relationship $\Delta$ $=1.76 k_{B} T_{C}$. Figure 3(c) shows spectral weight integrated 

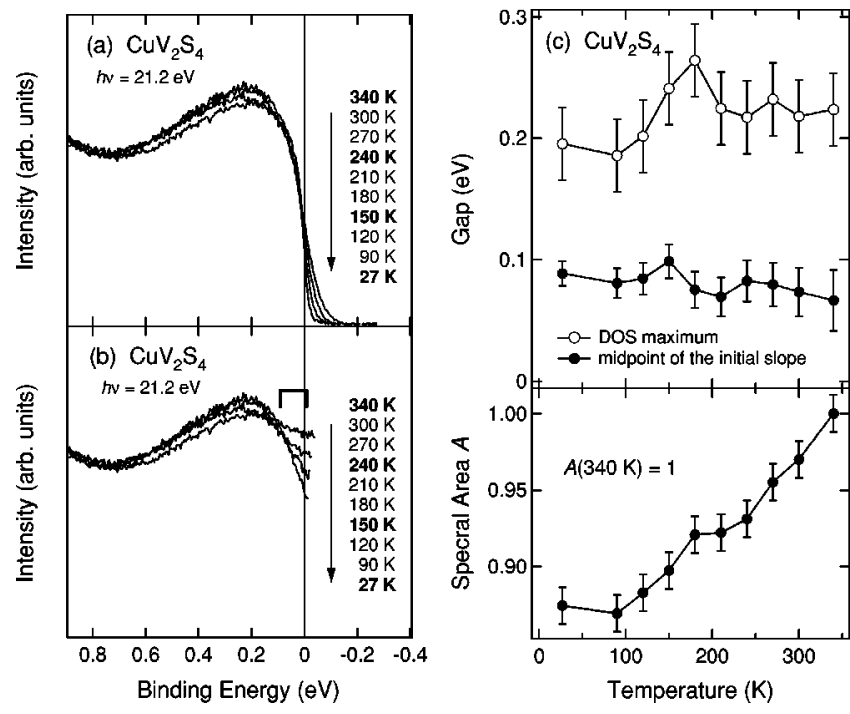

FIG. 3. (a) Detailed temperature dependence of photoemission spectra of $\mathrm{CuV}_{2} \mathrm{~S}_{4}$ near the Fermi level. The spectra have been normalized to the integrated intensity between $1.3 \mathrm{eV}$ and $E_{F}$. (b) Photoemission spectra with the Fermi-Dirac distribution divided out. (c) Magnitude of the gap estimated from the midpoint of the initial slope and the DOS maximum (upper panel) and the spectral weight integrated from $0.10 \mathrm{eV}$ to $E_{F}$ (lower panel) as functions of temperature.

from $0.10 \mathrm{eV}$ to $E_{F}$ at various temperatures. One can see that the temperature dependence is monotonous up to $>300 \mathrm{~K}$ and there is no clear change at the transition temperature of $90 \mathrm{~K}$ and below it. Those observations mean that the CDW transition in $\mathrm{CuV}_{2} \mathrm{~S}_{4}$ is beyond the mean-field behavior. In other words, the CDW formation is in the strong-coupling regime. The persistent pseudogap above $90 \mathrm{~K}$ may be due to short-range CDW formation driven by the strong electronphonon interaction. Following this interpretation, $T_{C}$ is described as the temperature at which a long-range CDW is formed.

Changes in the spectra with temperature in CDW systems were theoretically studied by Lee, Rice, and Anderson. ${ }^{17}$ They treated low-dimensional fluctuations within the meanfield theory and showed that increasing temperature causes the simultaneous occurrence of a decrease of the gap magnitude and spectral weight transfer from higher binding energies to lower binding energies. Our results do not agree with this prediction. Although there are no available theoretical results that can be directly compared with the present results at this stage, the spectral transfer over the wide energy range of $\sim 0.8 \mathrm{eV}$ suggests that electron correlation among $\mathrm{V} 3 d$ orbitals may also be important.

Recently, the SDW in Cr was studied by means of angleresolved photoemission spectroscopy (ARPES). ${ }^{18}$ It was reported that an energy gap of $2 \Delta \sim 200 \mathrm{meV}$ opened along the $\Gamma-S$ line in the Brillouin zone at room temperature and the SDW transition temperature was $440 \mathrm{~K}$. These values are somewhat enhanced from those of bulk $\mathrm{Cr}$ due to the increase of the magnetic moment at the surface. In our case, if the electron-phonon coupling is enhanced at the surface, the CDW transition temperature may increase as well. However, the observed discrepancy between the experiment and BCS theory is so large that the unusual temperature dependence may not be explained only by the surface effects. In order to see whether the partial nesting scenario is applicable or not, ARPES measurements of $\mathrm{CuV}_{2} \mathrm{~S}_{4}$ are strongly desired. In spite of the difficulty arising from the three dimensionality, it is important to settle the anisotropy of the gap opening behavior in $\mathrm{CuV}_{2} \mathrm{~S}_{4}$.

\section{B. Band-structure calculation}

In order to see whether the partial nesting scenario is valid or not, we have performed first-principles band-structure calculations on $\mathrm{CuV}_{2} \mathrm{~S}_{4}$ and examined the possibility of CDW formation from the viewpoint of one-electron band theory. We have calculated the bare electronic susceptibility defined as follows:

$$
\chi(\mathbf{q})=\sum_{\mu \nu} \chi_{\mu \nu}(\mathbf{q})=-2 \sum_{\mu \nu} \sum_{\mathbf{k}} \frac{f\left(\epsilon_{\mathbf{k}+\mathbf{q}}^{\nu}\right)-f\left(\epsilon_{\mathbf{k}}^{\mu}\right)}{\epsilon_{\mathbf{k}+\mathbf{q}}^{\nu}-\epsilon_{\mathbf{k}}^{\mu}},
$$

where $f(\epsilon)$ is the Fermi-Dirac distribution function, $\mu$ and $\nu$ are band indices, and $\epsilon_{\mathbf{k}+\mathbf{q}}^{\nu}$ and $\epsilon_{\mathbf{k}}^{\mu}$ are band energies at points separated by the wave vector $\mathbf{q}$. It is generally believed that peaks in $\chi(\mathbf{q})$ are important in CDW formation and in fact they can explain Kohn-type anomalies in phonon spectra of Sc. ${ }^{19}$ The present band-structure calculation for $\mathrm{CuV}_{2} \mathrm{~S}_{4}$ has been carried out in the local-density approximation (LDA) using a full-potential, scalar-relativistic implementation ${ }^{20}$ of the linear augmented-plane-wave (LAPW) method. ${ }^{21}$ The cutoff has been set at values such that the calculation include 60 LAPW's/atom (10-Ry plane-wave cutoff) and spherical harmonic terms up to $l=6$. The crystalline charge density and potential have been expanded with about 8900 plane waves (48-Ry cutoff) in the interstitial region and lattice harmonics with $l_{\max }=6$ within the muffin-tin spheres $\left(R_{\mathrm{Cu}}\right.$ $\approx 2.10$ a.u., $R_{\mathrm{V}} \approx 2.44$ a.u., $R_{\mathrm{S}} \approx 2.09$ a.u.). Brillouin-zone (BZ) integration has utilized a ten-point $\mathbf{k}$ samples in the $1 / 48$ irreducible wedge. Exchange and correlation effects in the LDA potential have been included via the Wigner interpolation formula. ${ }^{22}$ The atomic $\mathrm{Cu}\left(3 d^{10} 4 s^{1}\right), \mathrm{V}\left(3 d^{4} 4 s^{1}\right)$, and $\mathrm{S}\left(3 s^{2} 3 p^{4}\right)$ states were treated as valence electrons in this study whereas the more tightly bound levels were included via the frozen-core approximation. The calculation of the electronic susceptibility was made for $T=0$ by application of the tetrahedron method after Rath and Freeman. ${ }^{19} \mathrm{BZ}$ integration has been done based on the energy eigenvalues at $916 \mathbf{k}$ points in the irreducible wedge directly calculated using the LAPW method.

Figure 4 shows the intraband component of the calculated susceptibility $\chi_{\mu \mu}$ for the 45th and 46th bands. Although there are five bands which cross the Fermi level, we have considered the above two bands because they occupy more than $90 \%$ weight of the density of states at $E_{F}$. We also calculated the interband contribution $\chi_{\mu \nu}(\mu \neq \nu)$, but it has no well-defined structures and is therefore not shown in Fig. 4. The calculation was made along the high-symmetry lines 


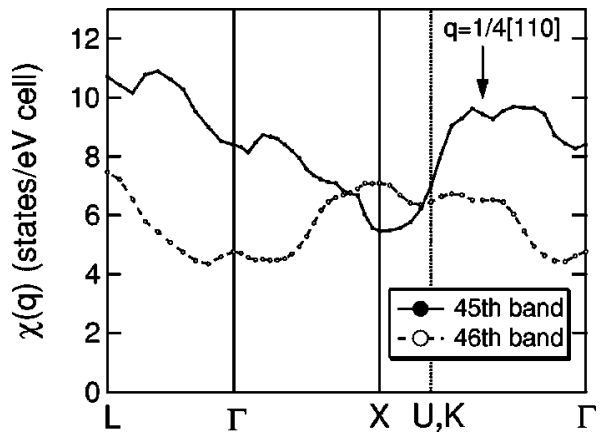

FIG. 4. Calculated susceptibility in $\mathrm{CuV}_{2} \mathrm{~S}_{4}$ for the 45th and 46th bands. The arrow shows a small peak corresponding to the observed superstructure modulation wave vector $\mathbf{q}$

in the BZ. One can easily see that there is no prominent structure for the 46th band, whereas there is a small peak for the 45 th band at $\mathbf{q}=\frac{11}{40}[110]$ along the $\Gamma-K$ line. This is consistent with the observed wave vector characterizing the CDW of $\mathbf{q} \sim \frac{1}{4}[110]$. In the case of chromium, a similar calculation was presented and the calculated peak along the $\Gamma$ $H$ line coincides with the experimental value of the SDW. ${ }^{23}$ We note that only a small peak in $\chi(\mathbf{q})$ leads to the actual SDW in Cr metal. In the case of $\mathrm{CuV}_{2} \mathrm{~S}_{4}$, the peak at $\mathbf{q}$ $\sim \frac{1}{4}[110]$ may also be relevant to the formation of the CDW in spite of its smallness. Thus the conventional CDW scenario, i.e., nesting of the Fermi surface, may be at least partly responsible for CDW formation from the viewpoint of the first-principles band-structure calculation.

\section{CONCLUSION}

We have observed that the photoemission spectra of $\mathrm{CuV}_{2} \mathrm{~S}_{4}$ change over a wide temperature range from $90 \mathrm{~K}$ to $340 \mathrm{~K}$. The spectra show pseudogap-like behavior and the gap size is estimated to be $\sim 90 \mathrm{meV}$. This pseudogap-like behavior suggests partial nesting of the Fermi surface. With increasing temperature, the pseudogap does not close but is filled by spectral weight transferred from high binding energies. Both the pseudogap and the spectral weight near $E_{F}$ do not show a clear change at the transition temperature of $90 \mathrm{~K}$ and below it. These observations do not follow the BCS behavior. The large energy scale of the pseudogap compared to the transition temperature suggests that the interaction involved is in the strong-coupling regime. We have performed first-principles band-structure calculations and have calculated the bare electronic susceptibility $\chi(\mathbf{q})$. We have found a peak in the electronic susceptibility at $\mathbf{q} \simeq \frac{11}{40}[110]$, which is consistent with the observed value of $\mathbf{q} \sim \frac{1}{4}[110]$ by $\mathrm{x}$-ray diffraction. This result suggests that partial nesting of the Fermi surface is indeed a possible mechanism for CDW formation.

\section{ACKNOWLEDGMENTS}

This work was supported by a Grant-in-Aid for Scientific Research on Priority Areas "Novel Quantum Phenomena in Transition-Metal Oxides" from the Ministry of Education, Culture, Sports, Science and Technology of Japan. J.M. acknowledges support from the Japan Society for the Promotion of Science for Young Scientists.
${ }^{1}$ R.M. Fleming, F.J. DiSalvo, R.J. Cava, and J.V. Waszczak, Phys. Rev. B 24, 2850 (1981).

${ }^{2}$ J. Mahy, D. Colaitis, D. van Dyck, and S. Amelinckx, J. Solid State Chem. 68, 320 (1987).

${ }^{3}$ Y. Yoshikawa, S. Wada, K. Miyatani, T. Tanaka, and M. Miyamoto, Phys. Rev. B 55, 74 (1997).

${ }^{4}$ T. Hagino, Y. Seki, S. Takayanagi, N. Wada, and S. Nagata, Phys. Rev. B 49, 6822 (1994).

${ }^{5}$ N. Le Nagard, A. Katty, G. Collin, O. Gorochov, and A. Willig, J. Solid State Chem. 27, 267 (1979).

${ }^{6}$ T. Ohno, Y. Kishimoto, and K. Miyatani, Physica B 230-232, 988 (1997).

${ }^{7}$ Y. Kishimoto, T. Ohno, T. Kanashiro, Y. Michihiro, K. Mizuno, M. Miyamoto, T. Tanaka, and K. Miyatani, Solid State Commun. 96, 23 (1995).

${ }^{8}$ T. Sekine, K. Uchinokura, H. Iimura, R. Yoshizaki, and E. Matsuura, Solid State Commun. 54, 187 (1984).

${ }^{9}$ Z.W. Lu, B.M. Klein, E.Z. Kurmaev, V.M. Cherkashenko, V.R. Galakhov, S.N. Shamin, Yu.M. Yarmoshenko, V.A. Trofimova, St. Uhlenbrock, M. Neumann, T. Furubayashi, T. Hagino, and S. Nagata, Phys. Rev. B 53, 9626 (1996).

${ }^{10}$ E. Fawcett, Rev. Mod. Phys. 60, 209 (1988).

${ }^{11}$ M. Grioni and J. Voit, in Electron Spectroscopies Applied to Low-
Dimensional Materials, edited by H. Stanberg and H. Hughes (Kluwer, Dordrecht, 2000).

${ }^{12}$ Ferromagnetic Materials: A handbook on the properties of magnetically ordered substances, edited by E. P. Wohlfarth (NorthHolland, Amsterdam, 1980), pp. 603-745.

${ }^{13}$ S. Shin, S. Suga, M. Taniguchi, M. Fujisawa, H. Kanzaki, A. Fujimori, H. Daimon, Y. Ueda, K. Kosuge, and S. Kachi, Phys. Rev. B 41, 4993 (1990).

${ }^{14}$ H.F. Franzen and G.A. Sawatzky, J. Solid State Chem. 15, 229 (1975).

${ }^{15}$ A. Fujimori, M. Saeki, and H. Nozaki, Phys. Rev. B 44, 163 (1991).

${ }^{16}$ T. Greber, T.J. Kreutz, and J. Osterwalder, Phys. Rev. Lett. 79, 4465 (1997).

${ }^{17}$ P.A. Lee, T.M. Rice, and P.W. Anderson, Phys. Rev. Lett. 31, 462 (1973).

${ }^{18}$ J. Schäfer, Eli Rotenberg, G. Meigs, S.D. Kevan, P. Blaha, and S. Hüfner, Phys. Rev. Lett. 83, 2069 (1999).

${ }^{19}$ J. Rath and A.J. Freeman, Phys. Rev. B 11, 2109 (1975).

${ }^{20}$ L.F. Mattheiss and D.R. Hamann, Phys. Rev. B 33, 823 (1986).

${ }^{21}$ O.K. Andersen, Phys. Rev. B 12, 3060 (1975).

${ }^{22}$ E. Wigner, Phys. Rev. B 46, 1002 (1934).

${ }^{23}$ C.G. Windsor, J. Phys. F: Met. Phys. 2, 742 (1972). 